\title{
Functional forgetting
}

DOI:

10.1038/nrn2183

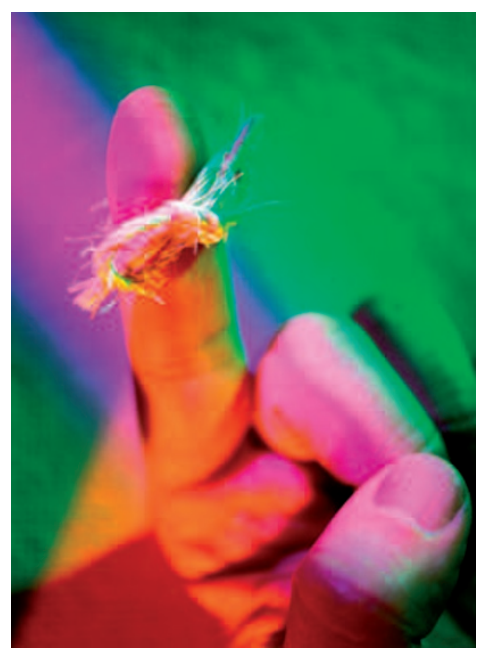

A familiar scenario: you use your new bank card to withdraw money, but when you are prompted to enter your PIN, you don't remember it. Instead, your old PIN numbers scroll through your mind. Competing memories require cognitive control mechanisms to identify relevant facts and suppress irrelevant information. Kuhl et al. now show that the cost of forgetting has the benefit of more efficient neural processing, improving one's memory for relevant information.

The study consisted of three phases. In the encoding phase, participants studied cue-target word pairs; each cue word had six associated target words. In the practice phase, participants were shown half of the cue words together with the first letter of half of the associated targets and asked to indicate whether or not they remembered the target word. This was repeated three times. Finally, in the memory phase, participants were shown all of the cue words and the first letter of all of the associated targets, and were tested on their memory for both the practiced and the unpracticed targets.

Unsurprisingly, in the practice phase the success rate improved between the first and third trials. In the memory phase, the practiced targets were the best remembered words. Interestingly, participants found it easier to forget unpracticed targets that were associated with practiced cues (competing targets) than unpractised targets that were associated with unpracticed cues, indicating that competition between words suppressed memory for the non-practiced word.

Using functional MRI scanning, the authors showed that in the practice phase there was higher activation in frontoparietal areas, including the anterior cingulate cortex (ACC) and prefrontal cortex (PFC), during successful retrievals as compared with unsuccessful retrievals. However, activation in the ACC and PFC, specifically in the ventro- and dorsolateral PFC (VLPFC and DLPFC, respectively), decreased from the first to the third successful trial, indicating a reduced demand on these regions as the unpracticed competing targets were forgotten. Indeed, decreased VLPFC and ACC activation correlated with increased forgetting of competing targets.

Participants who were good at suppressing competing targets (high suppressors) had more ACC activation during the first successful retrieval than low suppressors. Moreover, only high suppressors showed a subsequent decrease in ACC and right VLPFC activation, indicating that people who detect high mnemonic conflict resolve this by suppressing the competing memories, and that demand on the ACC and right VLPFC decreases accordingly.

By contrast, changes in DLPFC activation correlated with better memory for practiced target words rather than with better forgetting of non-practiced targets, indicating that this area might contribute to memory facilitation for relevant information rather than suppression of memory for competing information.

This study provided evidence that changing demands on the ACC and the right lateral PFC, which are involved in cognitive control, reflect memory suppression. It also showed that more efficient neural processing might be the benefit of forgetting.

Leonie Welberg

ORIGINAL RESEARCH PAPER Kuhl, B. et al. Decreased demands on cognitive control reveal the neural processing benefits of forgetting. Nature Neurosci. 3 June 2007 (doi:10.1038/nn1918) 